This is a self-archived version of an original article. This version may differ from the original in pagination and typographic details.

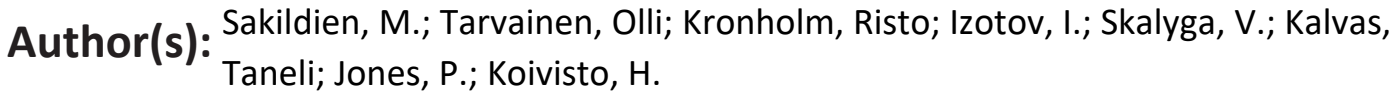

Title: Experimental evidence on microwave induced electron losses from ECRIS plasma

Year: 2018

Version: Published version

Copyright: @ AlP Publishing.

Rights: In Copyright

Rights url: http://rightsstatements.org/page/InC/1.0/?language=en

Please cite the original version:

Sakildien, M., Tarvainen, O., Kronholm, R., Izotov, I., Skalyga, V., Kalvas, T., Jones, P., \& Koivisto, $\mathrm{H}$. (2018). Experimental evidence on microwave induced electron losses from ECRIS plasma.

Physics of Plasmas, 25(6), Article 062502. https://doi.org/10.1063/1.5029443 


\section{Experimental evidence on microwave induced electron losses from ECRIS plasma}

M. Sakildien, O. Tarvainen, R. Kronholm, I. Izotov, V. Skalyga, T. Kalvas, P. Jones, and H. Koivisto

Citation: Physics of Plasmas 25, 062502 (2018); doi: 10.1063/1.5029443

View online: https://doi.org/10.1063/1.5029443

View Table of Contents: http://aip.scitation.org/toc/php/25/6

Published by the American Institute of Physics

\section{Articles you may be interested in}

Direct measurement of the transition from edge to core power coupling in a light-ion helicon source

Physics of Plasmas 25, 052101 (2018); 10.1063/1.5023924

Observation of high-temperature bubbles in an ECR plasma

Physics of Plasmas 25, 052113 (2018); 10.1063/1.5027588

$\mathrm{E} \times \mathrm{B}$ electron drift instability in Hall thrusters: Particle-in-cell simulations vs. theory

Physics of Plasmas 25, 061204 (2018); 10.1063/1.5017033

Characterization of xenon ion and neutral interactions in a well-characterized experiment

Physics of Plasmas 25, 062108 (2018); 10.1063/1.5030464

A simple scaling law for the equation of state and the radial distribution functions calculated by densityfunctional theory molecular dynamics

Physics of Plasmas 25, 060702 (2018); 10.1063/1.5026045

Ion velocity analysis of rotating structures in a magnetic linear plasma device

Physics of Plasmas 25, 061203 (2018); 10.1063/1.5019448

\section{PHYSICS TODAY}

MANAGER'S GUIDE

WHITEPAPERS

\section{READ NOW}

PRESENTED BY

Accelerate R\&D with Multiphysics Simulation
○OMSOL 


\title{
Experimental evidence on microwave induced electron losses from ECRIS plasma
}

\author{
M. Sakildien, ${ }^{1, a)}$ O. Tarvainen, ${ }^{2}$ R. Kronholm, ${ }^{2}$ I. Izotov, ${ }^{3}$ V. Skalyga, ${ }^{3}$ T. Kalvas, ${ }^{2}$ P. Jones, ${ }^{4}$ \\ and $\mathrm{H}$. Koivisto ${ }^{2}$ \\ ${ }^{1}$ Accelerator Department, iThemba LABS (Laboratory for Accelerator Based Sciences), PO Box 722 , \\ Somerset West 7192, South Africa \\ ${ }^{2}$ Department of Physics, University of Jyväskylä, 40500 Jyväskylä, Finland \\ ${ }^{3}$ Institute of Applied Physics, RAS, 46 Ul'yanova st., 603950 Nizhny Novgorod, Russian Federation \\ ${ }^{4}$ Subatomic Physics Department, iThemba LABS (Laboratory for Accelerator Based Sciences), PO Box 722, \\ Somerset West 7192, South Africa
}

(Received 14 March 2018; accepted 18 May 2018; published online 5 June 2018)

The balance between warm and hot $(>1 \mathrm{keV})$ electron density and their losses from the magnetic confinement system of an Electron Cyclotron Resonance Ion Source (ECRIS) plasma is considered to be one of the main factors determining the rate of the high charge state ion production. One of the key loss channels for heated electrons is thought to be induced by the injected microwaves. While this loss mechanism, referred to as rf-induced pitch angle scattering, has been studied theoretically and with computational tools, direct experimental evidence of its significance in minimum-B ECRIS plasmas remains limited. In this work, experimental evidence of microwave induced electron losses in the axial direction is presented in both continuous wave $(\mathrm{CW})$ and pulsed operation of a $14 \mathrm{GHz}$ ECRIS. In the $\mathrm{CW}$ mode, the experiment was carried out by comparing the characteristic X-ray emission from the plasma volume and from the surface of the biased disc located in the flux of the escaping electron at the axial magnetic mirror. Parametric sweeps of magnetic field, neutral gas pressure, and microwave power were conducted to determine their effect on electron losses. In the pulsed mode, the experiment was conducted by measuring the flux of escaping electrons through aluminum foils of different thicknesses providing some energy resolution. Both diagnostics support the view that rf-induced losses account for up to $70 \%$ of total hot electron losses and their importance depends on the source parameters, especially power and neutral gas pressure. Published by AIP Publishing. https://doi.org/10.1063/1.5029443

\section{INTRODUCTION}

The extractable charge state distribution (CSD) of ions from an Electron Cyclotron Resonance Ion Source (ECRIS), ${ }^{1}$ as well as the plasma energy confinement time, is strongly dependent on the density of the different electron populations (see Barué et $a l^{2}$ and Shirkov ${ }^{3}$ ) present inside the ECRIS plasma. ${ }^{4}$ The number of warm and hot electrons lost from the magnetic confinement system of an ECRIS is therefore a critical parameter affecting the volumetric production rate of high charge state ions. Additionally, the warm and hot electrons are thought to play a crucial role in the build-up of an electrostatic potential which is suggested to regulate ion confinement via a small potential dip in the core of the plasma. This confinement scheme, extensively discussed by Petty et al., ${ }^{5}$ enables ions to remain in the plasma for a sufficiently long time in order to reach the desired charge state through step-wise electron impact ionization.

Some of the first theoretical studies on electron cyclotron resonance heating (ECRH) in magnetic mirror devices were published in the $1970 \mathrm{~s},{ }^{6}$ with the relativistic theory of ECRH developed later. ${ }^{7}$ In the 1980s, Mauel performed experimental measurements on ECRH of a highly ionized

\footnotetext{
${ }^{\text {a)} E l e c t r o n i c ~ m a i l: ~ m u n e e r @ t l a b s . a c . z a ~}$
}

plasma in a pulsed magnetic mirror configuration. ${ }^{8}$ The results obtained in this study demonstrated that the fractional densities of the warm and hot electron populations depend on the absorbed microwave power. The experiment revealed an enhanced electron end-loss current associated with the increase in microwave power and consequently it was concluded that the electromagnetic (EM)-field of the incident microwaves could enhance electron losses. Moreover, an investigation of helium plasma diamagnetism and electron end losses conducted by Perret et al. ${ }^{4}$ suggested that the warm and hot electron losses from the magnetic confinement system of an ECRIS plasma can under certain conditions exceed those accounted for by Coulomb collisions only. This led to the suggestion of enhanced electron losses induced by the EM-field of the incident microwave power affecting the performance of modern ECR ion sources. The role of microwave induced pitch angle scattering in ECRIS was recently discussed for e.g., by Noland et al. comparing the emission power from the plasma bremsstrahlung to the wall bremsstrahlung ${ }^{9}$ and by Cluggish et al. conducting a computational study on the topic. ${ }^{10}$

Although the concept of radiofrequency (rf)-induced pitch angle scattering is well-established, direct and systematic experimental evidence on its role in ECRIS plasmas is scarce. With the current investigation, three complementary plasma diagnostic methods are combined to study electron 
losses from the minimum-B confinement system of an ECRIS. The diagnostics study different electron populations of the electron energy distribution function (EEDF) allowing for a more complete understanding of the physics governing electron losses. For the first part of the investigation, $\mathrm{K} \alpha$ diagnostics obtained in the continuous wave $(\mathrm{CW})$ mode are used to estimate the relative electron losses from the magnetic confinement system. The electron losses are estimated from the ratio of the thick target $\mathrm{K} \alpha$ (iron) emission from the axial wall of the plasma confinement vessel to the $\mathrm{K} \alpha$ emission of the confined ion species (argon). The measurement of this ratio as a function of the ion source parameters probes the electron loss rate against the inner shell ionization rate, which is affected by mostly the warm electron density, and thus can be used for identifying electron loss mechanisms in action. With the second part of the investigation, bremsstrahlung diagnostics and electron end-loss current are measured in pulsed mode operation. This allows a comparison of the electron losses between microwave power on and off periods and sheds new light on the influence of the plasma parameters on electron losses. The electron end-loss current is measured through different attenuators (aluminium foils) enabling a study of the electron losses as a function of electron energy with discrete steps. Altogether, the investigation demonstrates the feasibility of the $\mathrm{K} \alpha$-diagnostics for probing the role of microwave induced electron losses in $\mathrm{CW}$ mode while the pulsed experiments provide direct evidence of such losses in ECRIS plasmas.

\section{THEORETICAL BACKGROUND}

\section{A. Electron loss processes}

The electrons inside an ECRIS are confined by a complex magnetic field. This magnetic field consists of a solenoid mirror field, for axial confinement, combined with a multipole field, for radial confinement, forming the so-called minimum-B field and magnetic bottle. ${ }^{1}$ In the absence of collisions and interaction with electromagnetic waves, the confinement of an electron depends only on its parallel $\left(v_{\|}\right)$and perpendicular $\left(v_{\perp}\right)$ velocity components defining the electron loss cone in velocity space. ${ }^{1}$ Upon entering the loss cone, the electrons experience a force as a result of the gradient and curvature of the magnetic field and will leak out of the magnetic bottle as thoroughly discussed by Thuillier et al. ${ }^{11}$ From the analysis performed, it appears that the electron losses through the weakest mirrors occur faster in comparison to the characteristic time of the diffusion into the loss cone $^{11}$ i.e., the loss cone can be assumed to be empty. Contrary to the plasma electrons, high charge state ions are confined electrostatically ${ }^{5,12}$ and their losses are due to electron losses i.e., ions follow the electrons owing to the ambipolar field. ${ }^{13}$

The (electron) loss cone can be populated by a number of processes, as thoroughly discussed in the literature, ${ }^{10,14}$ including Coulomb collisions, inelastic collisions, microwave induced pitch angle scattering, and non-linear effects. ${ }^{15}$ The most important loss channels in stable operating conditions of highperformance ECRISs are considered to be collisional and microwave induced losses, the latter being discussed and studied extensively in this paper. Additional loss mechanisms include synchrotron radiation losses ${ }^{7}$ and radial diffusion $\operatorname{losses}^{16}$ but their contributions on the total flux of escaping electrons are considered to be insignificant. ${ }^{10}$

\section{B. Microwave induced electron losses from the ECRIS plasma}

In ECR plasmas, the electrons interact with the EM-field of the microwaves mainly at a surface where the resonance condition given by

$$
\omega_{r f}=\omega_{c e}(B)-k_{\|} v_{\|}
$$

is fulfilled. Here, $\omega_{r f}$ is the microwave (angular) frequency, $k_{\|}$is its parallel wave number, and $\omega_{c e}(B)$ is the relativistic electron gyrofrequency at magnetic field $B$. For the purpose of this study, it is sufficient to consider only the interaction between the circulating electrons and the right-hand circularly polarized wave (RHCP) component. Considering energy conservation in the frame moving with the wave, it can be shown that the electrons interacting with the RHCP wave, of phase velocity $v_{p}$, move in the velocity space along a circle defined by

$$
v_{\perp}^{2}+\left(v_{\|}-v_{p}\right)^{2}=a^{2},
$$

where $v_{\perp}$ and $v_{\|}$are the electron velocity components perpendicular and parallel to the external magnetic field, respectively, and $a$ is a constant. ${ }^{17}$ It is convenient to express this in a spherical polar coordinate system $(v, \psi, \phi)$ in the velocity space, where $v$ is the electron speed, $\arccos \psi$ is the pitch angle, i.e., $\psi=\frac{v_{\|}}{v}$, and $\phi$ is the angle about the axial magnetic field. ${ }^{4}$ The equation defining the loss cone then becomes

$$
v^{2}-2 v \psi v_{p}=b^{2}
$$

where $b$ is yet another constant. Differentiating Eq. (3) yields the increments $(\Delta v, \Delta \psi)$ in velocity and pitch angle during the interaction, i.e.,

$$
\Delta v\left(\frac{v}{v_{p}}-\psi\right)-v \Delta \psi=0 .
$$

In order to understand microwave induced loss processes, we need to consider two cases defined by the phase difference between the electron gyromotion and the electric field of the microwaves i.e., (a) $\Delta v>0$ and (b) $\Delta v<0$, corresponding to acceleration or deceleration of electrons at the resonance, respectively.

1. Electron energy gain $(\Delta v>0)$ : it follows from Eq. (4) that if $\left(\frac{v}{v_{p}}-\psi\right)<0$, then $\Delta \psi<0$, similarly if $\left(\frac{v}{v_{p}}-\psi\right)$ $>0$, then $\Delta \psi>0$. We may rewrite this condition as $\frac{v}{v_{p}} \lessgtr \frac{v_{\|}}{v}$. It is evident from $v^{2}=v_{\|}^{2}+v_{\perp}^{2}$ that $\frac{v_{\|}}{v} \leqslant 1$ and hence the condition for microwave heating improving electron confinement (decrease of $\psi$ ) becomes $\frac{v}{v_{p}}<1$ i.e., $v<v_{p}$. On the contrary, microwave heating induces electron losses (increase of $\psi$ ) if $\frac{v}{v_{p}}>1$ i.e., $v>v_{p}$. Since 
electrons with velocities above the phase velocity are mainly pitch-angle scattered, the maximum mean velocity of the electron distribution function is the phase velocity at the resonance.

2. Electron energy loss $(\Delta v<0)$ : applying similar reasoning as above, it follows from Eq. (4) that $\Delta \psi \lessgtr 0$ when $v \gtrless v_{p}$ i.e., for electron velocities less than the phase velocity of the EM-wave, the electron confinement is weakened by the interaction between electrons and the microwave electric field in the decelerating phase. On the other hand, electrons whose velocity exceeds the phase velocity become better confined as a result of interacting with the magnetic field of the EM-wave despite their energy loss.

All of the interaction mechanisms identified above play a role in ECRIS plasmas. However, the improvement of the electron confinement due to pitch angle scattering $(\Delta v$, $\Delta \psi<0$ ) during electron deceleration is of negligible importance since, as explained above, the electrons exit the plasma through the weakest mirror very fast.

Following Girard et al., the mean velocity kick parallel $\Delta v_{\|}$and perpendicular $\Delta v_{\perp}$ to the magnetic field in a single resonance crossing can be expressed as ${ }^{18}$

$$
\begin{gathered}
\Delta v_{\|}=\frac{e E}{m_{e}} \frac{v_{\perp}}{v_{p}} \sqrt{\frac{\pi}{\omega^{\prime} v_{\|}}} \\
\Delta v_{\perp}=\frac{e E}{m_{e}}\left(1-\frac{v_{\perp}}{v_{p}}\right) \sqrt{\frac{\pi}{\omega^{\prime} v_{\|}}}
\end{gathered}
$$

where $-e$ and $m_{e}$ is the charge and mass (relativistic if necessary) of an electron, respectively, $E$ is the microwave electric field amplitude at resonance, and $\omega^{\prime}$ is the derivative of the local electron cyclotron angular frequency along the magnetic field $B$.

According to this formulation, the electron confinement is improved, i.e., $\frac{\Delta v_{\|}}{\Delta v_{\perp}} \leq 1$ when $v_{p} \geq 2 v_{\perp}$. This results to a stochastic diffusion coefficient $D_{\mu \mu}$ in the pitch angle which can be defined as ${ }^{14}$

$$
D_{\mu \mu}=\pi\left(\frac{e E}{2 m_{e}}\right)^{2} \frac{d}{L \omega}\left(\frac{v}{v_{p}}\right)^{2}=D_{v v}\left(\frac{v}{v_{p}}\right)^{2},
$$

where $d$ is the characteristic length for the magnetic field gradient, $L$ is the characteristic plasma length, and $\omega$ is the microwave (angular) frequency. $D_{v v}$ corresponds to the velocity diffusion coefficient. It is emphasized that the rfinduced pitch angle diffusion coefficient in Eq. (7) is less than the velocity diffusion coefficient if $v<v_{p}$ and increases quadratically with increasing ratio of the electron velocity to phase velocity. Furthermore, the diffusion coefficient is sensitive to the microwave electric field i.e., it can be expected that the rate of pitch angle scattering depends predominantly on the microwave power and plasma density (neutral gas pressure).

Estimating the phase velocity $v_{p}=\frac{\omega}{k}$, where $k$ is the wave number, in ECRIS plasmas, is challenging due to the inhomogeneous plasma density and the existence of the electron cyclotron resonance, which affect the dispersion relation of the EM-wave through the local plasma permittivity. According to Stix, ${ }^{16}$ the phase velocity can be expressed as

$$
\frac{v_{p}}{c}=\frac{1}{\pi^{\frac{1}{6}}}\left(\frac{\omega}{\omega_{p e}}\right)^{\frac{2}{3}}\left(\frac{\langle v\rangle}{c}\right)^{\frac{1}{3}}
$$

where $\omega_{p e}$ is the plasma oscillation frequency, $c$ is the velocity of light in vacuum, and $\langle v\rangle$ is the mean velocity of the (non-Maxwellian) electron distribution. The electron density in ECRIS plasmas is considered to be below the critical density at which $\omega=\omega_{p e}$, which implies that only $>260 \mathrm{keV}$ electrons satisfy the condition $v>v_{p}$ for pitch angle scattering. However, the given expression does not take into account the reduction of the phase velocity in the resonance, which is thoroughly discussed by Ropponen et $a l^{20}$ and Williamson et al. ${ }^{21}$ The actual phase velocity at the resonance depends for e.g., on the plasma density and is reduced at least by an order of magnitude ${ }^{20}$ from the maximum value given by Eq. (8), which implies that the warm and hot electron populations of ECRIS plasmas with energies on the order of $10-100 \mathrm{keV}$ are affected by microwave induced pitch angle scattering. These considerations demonstrate that although, due to stochasticity, on average ECR-heating improves the electron confinement, microwave EM-fields also cause electrons to populate the loss cone throughout their energy distribution especially at high plasma densities which implies that the pitch angle scattering can limit the densities of the warm and hot electron populations. ${ }^{4}$

\section{EXPERIMENTAL SETUP AND PROCEDURE}

The experimental data described in this work were taken with the JYFL $14 \mathrm{GHz}$ ECRIS, presented in Fig. 1 and described in detail by Koivisto et $a .^{22}$ The source has a plasma chamber length and a diameter of $28 \mathrm{~cm}$ and $7.8 \mathrm{~cm}$, respectively, and uses an Nd-Fe-B permanent magnet sextupole arrangement and solenoid coils forming a minimum-B structure for plasma confinement. The magnetic confinement field provided by the solenoid coils and sextupole, results in typical magnetic field strengths at injection and extraction of 2.0 $\mathrm{T}$ and $0.9 \mathrm{~T}$, respectively. The strength of the sextupole field on the wall of the plasma chamber is $1.09 \mathrm{~T}$ on the magnetic poles and $0.71 \mathrm{~T}$ in between them when the solenoids are not energized. The (typical) strength of the B-field and sextupole, as a function of axial and radial distance, respectively, is shown in the inset of Fig. 1. The plasma is sustained by $50-800 \mathrm{~W}$ of microwave power at $14.1 \mathrm{GHz}$. Typical operating neutral pressures are in the $10^{-7}$ mbar range.

The experimental setup used for the $\mathrm{K} \alpha$ diagnostic measurement campaign was thoroughly described by Sakildien et $a .^{23}$ and only the most important features are mentioned here. The axially emitted $\mathrm{K} \alpha \mathrm{X}$-rays are measured with a silicon drift detector (SDD) mounted in one of the ports of the vacuum chamber of the analyzing magnet [see Fig. 1(a)] providing a direct line-of-sight into the ECRIS plasma through the $\phi=8 \mathrm{~mm}$ extraction aperture. In an attempt to limit the measured spectrum to mostly observe the plasma volume and biased disc, a collimator structure $(\phi 900 \mu \mathrm{m})$ was installed in front of the entrance of the detector. A permanent 
(a) Characteristic X-ray measurement setup (CW operation)

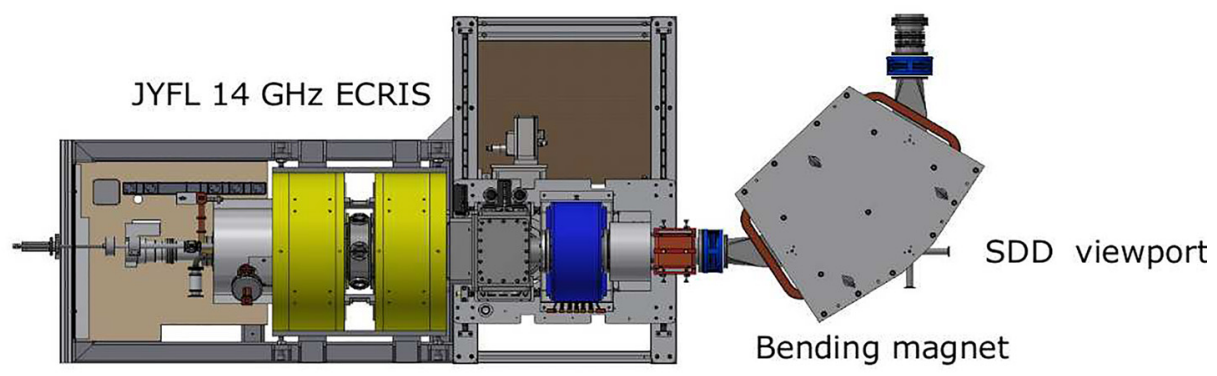

(b) Electron loss measurement setup (pulsed operation)

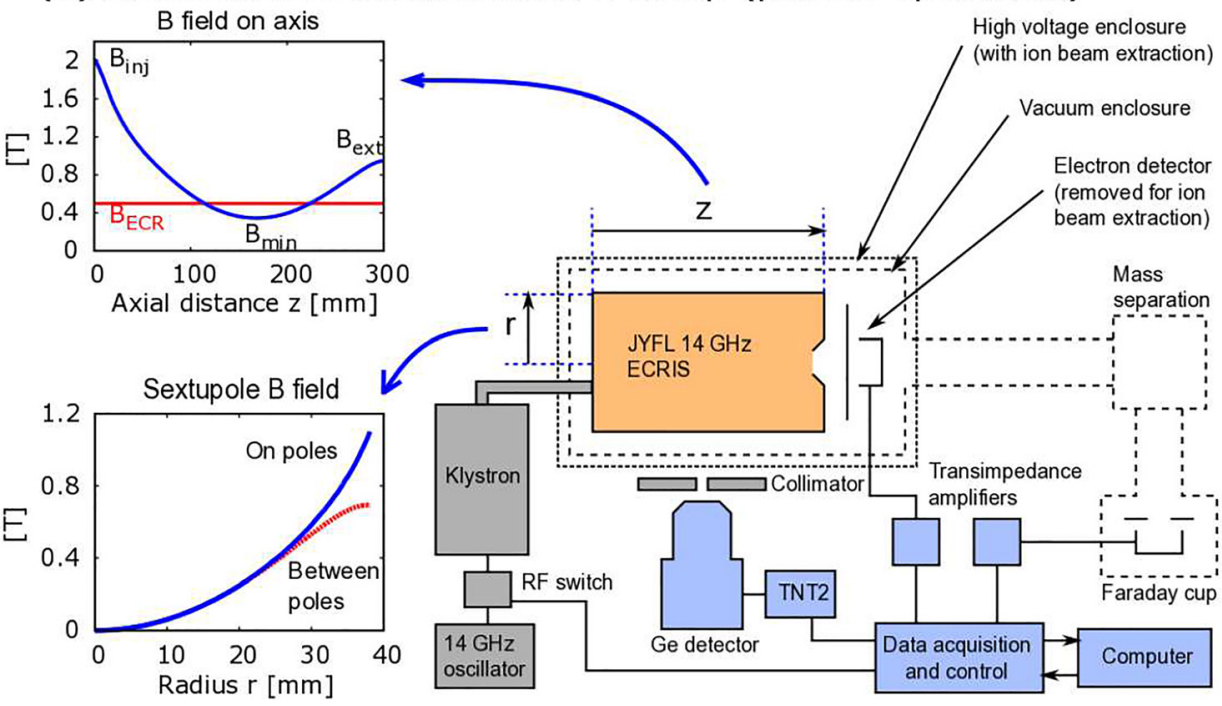

FIG. 1. Experimental setup used for measuring the relative electron losses in CW operation (a) and the setup used for measuring the escaping electrons in pulsed operation (b). magnet filter was also included in the experimental setup to protect the detector against damage by charged particles in the event the analyzing magnet is not energized. The detector has an energy resolution of $120 \mathrm{eV}$ at the $\mathrm{K} \alpha$ of $\mathrm{Mn}$ at $5.90 \mathrm{keV}$, which enables it to resolve many of the characteristic lines observed in a typical X-ray spectrum measured from the ECRIS plasma. To quantify the relative electron losses, the intensity of the $\mathrm{K} \alpha$ counts of the confined $\mathrm{Ar}$ ion species was compared to the intensity of the $K \alpha$ counts of Fe. The latter signal results from warm and hot electrons escaping the magnetic confinement system and interacting with the stainless steel biased disc located at the tip of the iron plug at the injection side of the plasma chamber. The argon counts measured by the detector emanate from the plasma volume and therefore represents the confined warm and hot electrons. This assertion is based on the fact that argon, as a noble gas, does not accumulate on the walls of the plasma chamber and is therefore confined to the plasma volume. The density of iron sputtered from the biased disc in the plasma volume, on the other hand, is negligible. This claim is supported by the fact that the iron extracted beam intensity is below the detection threshold of the Faraday cup in the transfer beamline. The characteristic emission of iron is therefore dominated by thick target (characteristic) radiation from the surface of the biased disc and the count rate of $\mathrm{Fe} \mathrm{K} \alpha$ is therefore proportional to the axial electron loss rate.

The schematic layout of the experimental setup used for measuring the electron losses in the pulsed mode is shown in
Fig. 1(b). The incident microwave power into the source was controlled with a fast RF-switch. A dedicated detector recording the electron current escaping the magnetic confinement through the extraction aperture was used for the experiments. The detector, first described by Izotov et al., ${ }^{24}$ consists of an array of aluminium foils, a suppression electrode, and a Faraday cup. A transimpedance amplifier with $40 \mu$ s rise-time (0\%-90\%) was used to convert the electron current to a voltage signal. The aluminium foils were assembled on a movable cradle whose position could be adjusted through a vacuum feed-through. Foils of specific thickness were used for the experiments to provide a crude energy resolution for the detection of escaping electrons. The thicknesses and corresponding (approximate) energy thresholds ${ }^{25}$ for the perpendicular angle of incidence are listed in Table I. The electron detector was positioned $205 \mathrm{~mm}$ downstream from the

TABLE I. Aluminium foil thickness and corresponding (approximate) energy threshold.

\begin{tabular}{lcc}
\hline \hline Foil number & $\begin{array}{c}\text { Thickness } \\
\left(\mathrm{mg} / \mathrm{cm}^{2}\right)\end{array}$ & $\begin{array}{c}\text { Energy threshold } \\
(\mathrm{keV})\end{array}$ \\
\hline 1 & No foil & 0 \\
2 & 1.0 & 20 \\
3 & 6.8 & 55 \\
4 & 18.4 & 100 \\
5 & 88.1 & 270 \\
6 & $270(1 \mathrm{~mm}$ plate $)$ & 570 \\
\hline \hline
\end{tabular}


extraction aperture (location of the foil) where the magnetic field strength has decreased to about 5\% compared to the extraction mirror. The effect of the magnetic field on electron trajectories and velocity components has been neglected when calculating the threshold energies in Table I. In order to install the electron detector, the pumping speed of the ion source had to be reduced from the nominal value. Consequently, all the data in this particular experiment were recorded using only residual gas (mainly oxygen and nitrogen) whose pressure remained constant at $4 \times 10^{-7}$ mbar throughout the experiments in the pulsed mode. Additionally, the high voltage was switched off (source potential was set to zero) during the pulsed mode measurements as this would have obstructed the escaping electron flux especially at low energies.

In order to understand the experimental conditions allowing the detection of pitch angle scattered electrons, some qualitative understanding of the sequence of events in the pulsed operation mode of an ECRIS is required. After switching off the microwaves, the electron density of the plasma decays exponentially due to collisional diffusion processes. Since the collision frequency of electrons scales with their temperature (energy) as $T_{e}^{-3 / 2}$, the hot electrons are the last ones to leak out from the magnetic bottle distorting the electron energy distribution during the afterglow plasma decays, which often results due to instabilities. ${ }^{24}$ These hot electrons act as seed electrons for the following pulse reducing the plasma breakdown time. ${ }^{15}$ The seed electron density naturally depends on the pulse pattern of the microwaves. At the very beginning of the microwave pulse, due to negligible plasma loading and high EM-fields, ${ }^{26}$ electrons interact strongly with the microwaves. Therefore, we expect to observe a burst of pitch angle scattered electrons from the plasma immediately after turning on the microwave power. It is also possible to study the role of microwaves inducing pitch angle scattering by measuring the decay characteristics of electron signals after switching off the microwave power. The microwave induced pitch angle scattering should manifest itself by abrupt drop of electron flux from the plasma as soon as microwave EM-fields have decayed.

In addition to direct measurement of electrons escaping through the extraction aperture, two auxiliary diagnostics monitoring the radially emitted bremsstrahlung $(>35 \mathrm{keV})$ and visible light emission were installed. The bremsstrahlung radiation was measured radially from one of the magnetic field poles of the ECR ion source simultaneously with the measurement of the escaping electron flux. The detector was located between the solenoids in the axial direction. Thus, the bremsstrahlung observed by the germanium detector is a mixture of thick target (wall) and plasma bremsstrahlung with the former dominating the spectrum. Because the focus of the experiments was to study the temporal evolution of the bremsstrahlung, no special attention was paid in optimizing the collimator structure. For this reason, only timeresolved (normalized) total count rates integrated over the energy range of the detected photons are presented. During the analysis, data from hundreds of rf pulses were combined to gain statistics. The counts caused by background radiation and pile-up events are subtracted from the data during the analysis with a dedicated code. ${ }^{20}$ Visible light was collected from the radial port (between the magnet poles) viewing the plasma with a lens system connected to a photodiode (IRD Inc. AXUVPN100) with an optical fiber. The photodiode was reverse biased with $24 \mathrm{~V}$ and the collected signal was amplified with a current to voltage amplifier (400 $\mu$ s risetime).

\section{EXPERIMENTAL RESULTS}

\section{A. Relative electron losses in CW mode of operation}

The relative electron losses from the magnetic confinement system of the ECRIS were studied in the CW mode by measuring the ratio of Fe-to-Ar $\mathrm{K} \alpha$ emission (denoted $r$ hereafter). Figure 2 shows a typical X-ray spectrum measured with the JYFL $14 \mathrm{GHz}$ ECRIS. The two dominant peaks i.e., $\mathrm{Ar} \mathrm{K} \alpha$ and $\mathrm{Fe} \mathrm{K} \alpha$ are identified in the figure. The remaining two prominent lines are $\mathrm{Al} \mathrm{K} \alpha(1.5 \mathrm{keV})$ and $\mathrm{Cr} \mathrm{K} \alpha$ $(5.4 \mathrm{keV})$. The detector efficiencies at the relevant energies is $0.91(\mathrm{Ar} \mathrm{K} \alpha)$ and $0.99(\mathrm{Fe} \mathrm{K} \alpha)$. The main sources of error for the relative electron loss results presented are related to the repeatability of the plasma conditions during the measurement and the variation of the plasma parameters over long data acquisition times. The uncertainty related to the repeatability was estimated from a set of consecutive measurements under identical conditions while the effect of long-term parameter drifts was minimized by reducing the data acquisition time to twenty minutes. Altogether, the experimental uncertainty is estimated to be $\pm 18 \%$. It is worth noting that the comparison of $\mathrm{Ar}$ and $\mathrm{Fe} \mathrm{K} \alpha$ count rate eliminates the error related to the detector alignment, the energy dependent efficiency, and the assumption of a spatially uniform and isotropic plasma distribution, which influence the accuracy of absolute measurements as discussed in Ref. 23

The experiments were started by conducting a parametric sweep of the four source tune parameters i.e., neutral gas pressure, microwave power, magnetic field configuration and biased disc voltage, and measuring $r$. These experiments revealed that the biased disc voltage had practically no influence on $r$ and hence the discussion is limited to the influence of the magnetic field configuration, microwave power, and neutral gas pressure.

Figure 3 shows the behaviour of $r$ as a function of the magnetic field strength, characterized by two figures; the $B_{\text {min }} / B_{E C R}$-ratio and the average field gradient parallel to

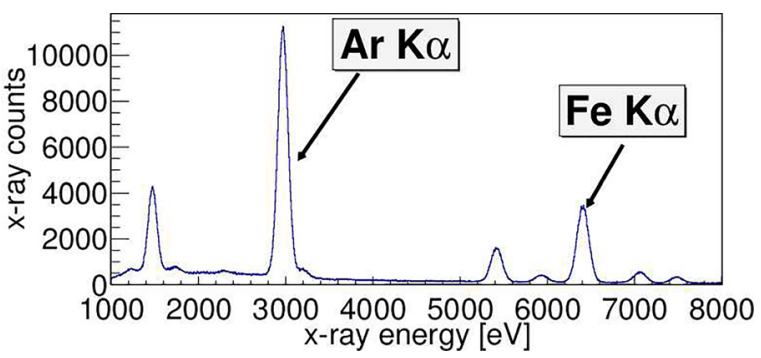

FIG. 2. A typical X-ray spectrum measured with the JYFL $14 \mathrm{GHz}$ ECRIS. With this spectrum, pile-up events have been removed, however the bremsstrahlung background has not been removed and the detector efficiency correction has not been applied. 


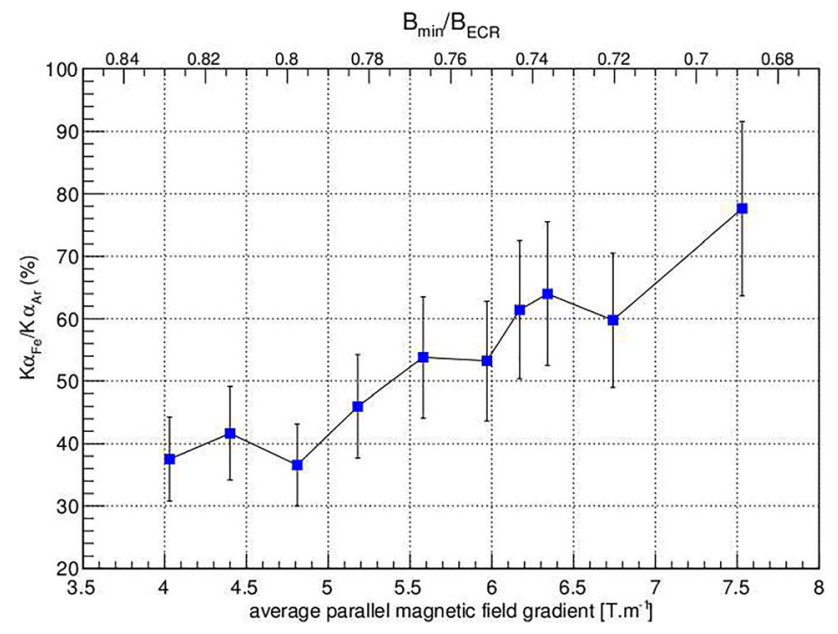

FIG. 3. Variation of $r$ as a function of the magnetic field strength with 400 $\mathrm{W}$ microwave power and $4.3 \times 10^{-7}$ mbar argon pressure.

the field, i.e., $\frac{\vec{B}}{|\vec{B}|} \cdot \nabla \vec{B}$. The magnetic field strength was altered by adjusting the currents of both coils simultaneously and hence preserving the relative strength of the axial magnetic mirrors. The fact that the JYFL $14 \mathrm{GHz}$ ECRIS has only two solenoids makes it impossible to adjust the $\mathrm{B}_{\text {min }}$ independently from the axial mirror fields. However, the mirror ratio is defined here as $\mathrm{B}_{\max } / \mathrm{B}_{E C R}$ (where max is injection or extraction), not $\mathrm{B}_{\max } / \mathrm{B}_{\min }$ which is often used for simple mirror traps. This definition is due to the fact that the magnetic confinement is based on the conservation of the magnetic moment which is violated each time the electron crosses the resonance where its velocity components are affected non-adiabatically. Hence, it is more meaningful to define the mirror ratio as $\mathrm{B}_{\max } / \mathrm{B}_{E C R}$, which is a common practice of the ECRIS community. The definition then implies that the change of the axial mirror field (injection and extraction) is directly proportional to the change of the mirror ratio, independent of $\mathrm{B}_{\min }$. The general trend observed from Fig. 3 is for the electron losses to decrease, indicated by the monotonic decrease in $r$, as $\mathrm{B}_{\text {min }} / \mathrm{B}_{E C R}$ increases or the average parallel magnetic field gradient decreases. It has been shown that the average electron energy, determined from bremsstrahlung diagnostics, is higher with increasing $\mathrm{B}_{\text {min }} / \mathrm{B}_{E C R}{ }^{19}$ Recent investigations performed by Izotov et al. also show directly that the local maxima of the EEDF of the escaping electrons shift towards higher energies with increasing $\mathrm{B}_{\text {min }} / \mathrm{B}_{E C R}$-ratio. ${ }^{27}$ It is therefore argued that the average electron energy increases with the decreasing average parallel magnetic field gradient. This relation can also be understood on the basis of more efficient ECR heating due to the lower gradient at the resonance surface. ${ }^{28,29}$ It is known that the inner shell ionization rate is a function of electron density, ion density, and rate coefficient for inner shell ionization. Accepting the fact that the ratio of the inner shell ionization rate of Fe-to-Ar should increase with increasing average electron energies ${ }^{30}$ leads to two possibilities to explain the experimental observation: (1) the electron density in the plasma volume is visible to the detector increases and/or (2) the electron loss rate towards the injection region decreases with increasing magnetic field strength i.e., $\mathrm{B}_{i n j}$ from $1.98 \mathrm{~T}$ to $2.13 \mathrm{~T}$ and $\mathrm{B}_{\text {ext }}$ from $0.90 \mathrm{~T}$ to $1.02 \mathrm{~T}$. The first possibility would cause $r$ to decrease as the Ar K $\alpha$ emission rate within the probed plasma volume would increase. This result is in line with the fact that the source performance, in terms of high charge state production, tends to increase with the B-field as long as the instability threshold is not crossed. ${ }^{15}$ However, this result of increasing electron density with increasing magnetic field strength is inconsistent with an earlier investigation which found that the electron density (responsible for inner shell ionization) remains virtually constant with the varying B-field in the range discussed here. ${ }^{23}$ This strengthens the argument that the result presented in Fig. 3 is most probably being caused by the axial B-field affecting the electron loss rate, i.e., the electron flux towards the biased disc is reduced when the axial magnetic field strength is increased. This would be due to the increasing injection (and extraction) mirror ratio reducing the axial electron losses. Thus, the conclusion drawn from the experimental observation in Fig. 3 is that the combined effect of higher plasma density (which is suggested by Sakildien et al. ${ }^{23}$ to play a lesser role) and reduced electron losses with increasing B-field results in the decrease in $r$. As remarked later again, the proposed reduced axial electron losses might affect the loss pattern of electrons and could most likely result in enhanced radial electron losses. Additionally, the variation of $r$ with the B-field sweep should be interpreted with caution. The B-field sweep can also change the EEDF of the escaping electrons as discussed earlier. ${ }^{27}$ However, the results presented in this work combined with an analysis of inner shell ionization rate coefficients indicate that the influence of the B-field strength on the axial flux of escaping electrons is greater than the influence of the shifting EEDF. ${ }^{27}$ It should also be pointed out that the effect of the B-field is not directly probing pitch angle scattering. Nevertheless, the results presented here demonstrate that $r$ is sensitive to particle losses and can therefore be used to probe the pitch angle scattering with different parametric sweeps. Furthermore, since it has now been established that $r$ is sensitive to the magnetic field strength, this parameter needs to be kept constant (as was done for the remainder of this investigation) during any experiments trying to probe the significance of microwave induced pitch angle scattering.

Figure 4 shows the variation of $r$ with neutral gas pressure at different microwave powers. The first observation made is that the neutral gas pressure has the most significant effect on the relative electron losses. This effect is especially pronounced at a low pressure. Based on auxiliary diagnostics signals (e.g., the biased disc current), it can be assumed that the electron density increases with increasing neutral pressure. The microwave power absorbed by the plasma at low neutral pressure/electron density results in an EEDF with high average electron energies. This is supported by the fact that the threshold magnetic field for the appearance of kinetic instabilities, driven by the anisotropy of the EEDF, is consistently lower at reduced neutral gas pressure. From an earlier investigation performed in the pulsed mode, it is known that the confinement time of high energy electrons can easily be of the order of seconds, ${ }^{31}$ when collisional scattering is the only loss process. The fact that the experimental 


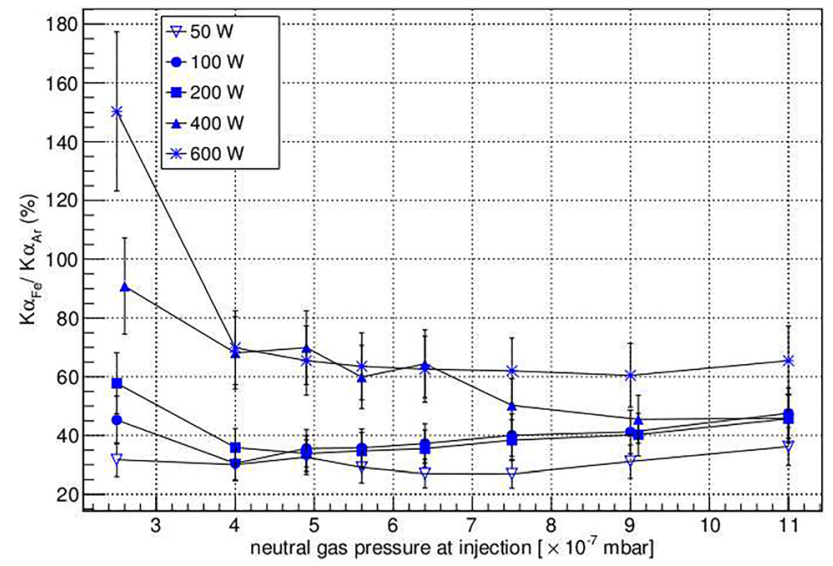

FIG. 4. Variation of $r$ as a function of neutral gas pressure at injection, at different microwave powers.

results in Fig. 4 indicate enhanced (warm and hot) electron losses at a low pressure point to a different loss mechanism at work. At low pressure/low plasma density, the EM-field strength is higher due to reduced damping of the field. Therefore, rf-induced scattering becomes more prominent at these conditions. This result is presumably displayed by the low pressure part of Fig. 4. The electron losses, as estimated by $r$, increase substantially if the incident microwave power increases. As the neutral gas pressure is increased, both the electron density and argon density increase. This could impact $r$ in two distinct ways: (1) the increasing argon density leads to increased argon inner shell ionization decreasing $r$ and/or (2) the increasing electron density leads the average electron energy to decrease as the absorbed microwave power is now distributed between a larger electron population and the strength of the EM-field at the resonance is damped. ${ }^{20}$ This results in a decrease in the mean velocity of the EEDF and reduced EM-field strength at the resonance due to increased damping of the EM-wave, reducing $r$ due to the reduced rate of rf-induced electron losses. Since these effects cannot be separated unambiguously, the conclusion drawn from the parametric sweep is that the best way to probe rf-induced scattering is to conduct a power sweep at several different neutral pressures keeping the magnetic field constant.

Figure 5 presents the variation of $r$ with absorbed microwave power, at different neutral gas pressures. The absorbed power is defined as the power available at the interface between the waveguide and the plasma (as discussed thoroughly in Ref. 23). The definition takes into consideration the power losses in the waveguide which are estimated to $\sim 20 \%$ as well as the reflected power which was measured to be less than $\sim 2 \%$. It is assumed that all power which is not lost in transmission or reflected back to the WR-62 waveguide is absorbed by the plasma i.e., losses at the chamber wall are not taken into account. Initially, $r$ increases with absorbed microwave power, which could be due to two instances: the changing EEDF favoring $r$ or alternatively (or simultaneously) there could be a loss mechanism i.e., pitch angle scattering proportional to the microwave power. The second tendency observed from the figure is a saturation of $r$

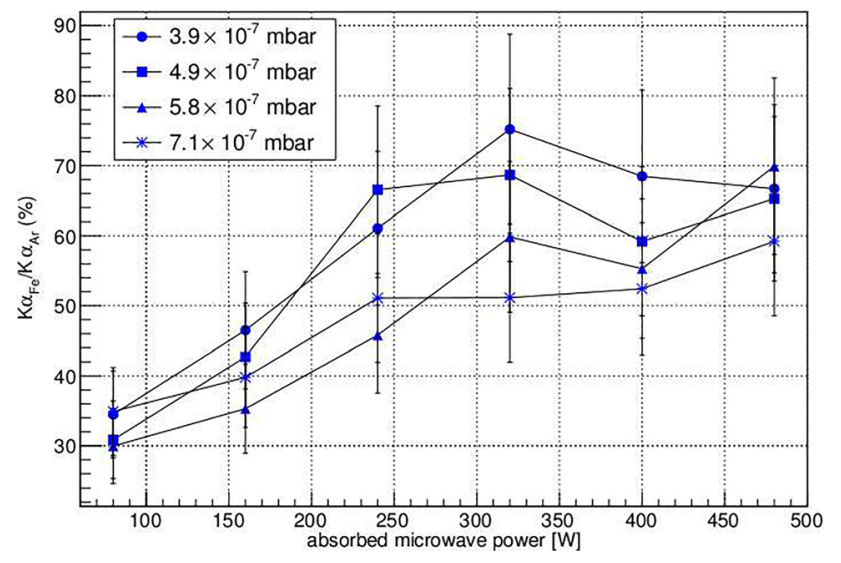

FIG. 5. Variation of $r$ as a function of absorbed microwave power, at different neutral gas pressures.

at powers higher than $\sim 300 \mathrm{~W}$. To account for both tendencies, the influence of the EEDF, electron density, and electron loss mechanisms on $r$ were considered. As a result of the increasing absorbed microwave power, the changing EEDF could result in both increased electron density as well as higher average electron energies. The increased electron density would result in both argon and iron $\mathrm{K} \alpha$ emission increase, under the assumption that the rates of the various electron loss mechanisms remain constant. This would not impact $r$ and therefore could not influence the first tendency observed in Fig. 5. The changing EEDF could also result in a higher average electron energy. This could propel the average electron energy pass the optimum required for inner shell ionization of argon and closer to the optimum for inner shell ionization of iron. The increasing average electron energy could also lead to enhanced electron losses due to pitch angle scattering. From the discussion in Sec. II, it is known that the rf induced velocity space diffusion of the electrons and their subsequent losses are driven by the relation between the mean velocity of the EEDF and the phase velocity of the EM-field. Increasing the mean velocity in excess of the phase velocity of the EM-field (in the case of electron heating) would induce increased electron losses driven by the microwave heating. These effects resulting from increasing average energy of the electrons could account, either partially or wholly, for the first tendency observed in Fig. 5. The variation of the EEDF, as a function of power is, however, not supported by the recent experimental data reported by Izotov et al. ${ }^{27}$ Thus, it seems fair to argue that purely based on elimination of the changing EEDF and increasing electron density, the measured result of increasing $r$ with microwave power is most likely due to the increased rate of pitch angle scattering. It is worth noting that Perret et al. arrived at a similar conclusion by assessing the results of a power sweep obtained with diagnostics complementary to this work. ${ }^{4}$ Assessing the second tendency, namely the saturation of $r$ at microwave powers $>300 \mathrm{~W}$, against the three parameters that influence $r$ leads to the conclusion that this tendency is dictated by the plasma energy content. The saturation of the plasma energy content with increasing microwave power has been observed e.g., by Noland et al. measuring the diamagnetic effect of the ECRIS plasma in the pulsed mode. ${ }^{9}$ From 
a previous investigation, it was observed that the $\arg$ on $\mathrm{K} \alpha$ emission rate saturates with increasing absorbed microwave power. $^{23}$ The current investigation also indicates that $r$ saturates with absorbed microwave power. Altogether, this leads to the conclusion that the iron $\mathrm{K} \alpha$ emission rate also saturates with the absorbed microwave power. It can be reasonably assumed that the increasing absorbed microwave power results in increasing electron density. Beyond a certain threshold, the absorbed microwave power becomes insufficient to heat the additional electrons resulting from ionization and causes the saturation of $r$ observed in Fig. 5 and discussed by Perret et al. ${ }^{4}$ Also, it can be argued that the EM-field of the microwave scales with the power $(P)$ as the field is proportional to $\sqrt{P}$ and is also affected by the plasma density through damping effects. Thus it is expected that the pitch angle scattering rate which depends on the EM-field strength does not follow the injected power in a linear manner.

\section{B. Electron losses in the pulsed operation mode}

This experimental campaign was started by measuring the temporal evolution of escaping electron signals with different aluminum foils. Different pulse patterns were used in order to vary the density of the so-called seed electrons. ${ }^{32}$ Figure 6 shows an example of the electron signals (in $\mu \mathrm{A}$ ) measured with $25 \mathrm{~ms} / 200 \mathrm{~W}$ microwave pulses at a repetition rate of $2 \mathrm{~Hz}$ (note different vertical scales). In the given example, the solenoid magnetic field (injection/minimum/ extraction) was set to $B_{\text {inj }}=2.11 \mathrm{~T} / \mathrm{B}_{\min }=0.39 \mathrm{~T} / \mathrm{B}_{\text {ext }}$ $=1.02 \mathrm{~T}$ (later referred as the B-field profile \#3).

The data in Fig. 6 reveal that turning on the microwave power leads to a burst of electrons from the trap. In this example, the duration of the transient is about $100 \mu \mathrm{s}$. The characteristics of the ignition peak, i.e., duration and magnitude, were observed to change with the ion source settings. The given example was selected for display because depending on the repetition rate, the peak either vanishes (lack of

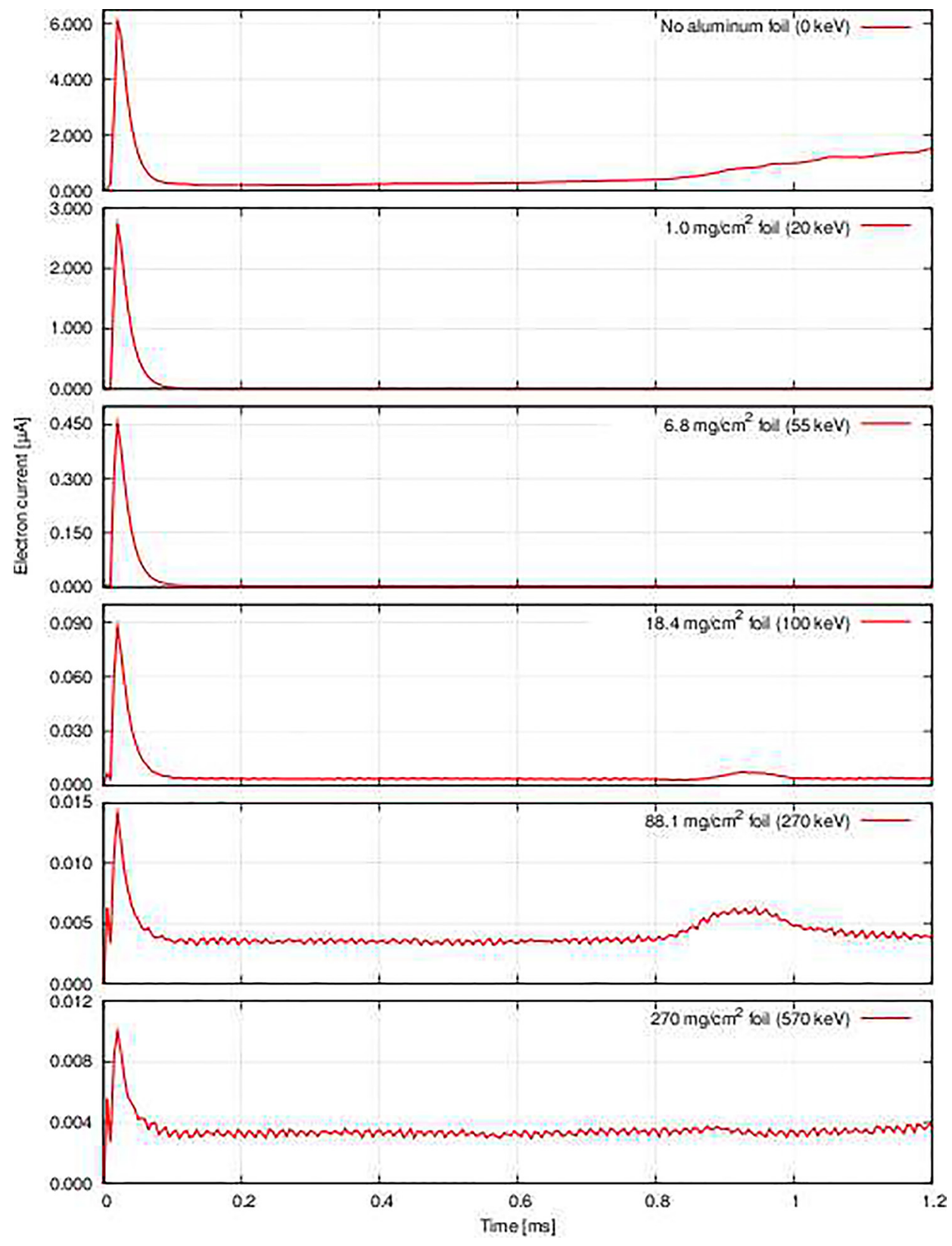

FIG. 6. Variation of the electron signal with a $25 \mathrm{~ms} / 200 \mathrm{~W}$ microwave pulse at a repetition rate of $2 \mathrm{~Hz}$ at $\mathrm{B}$-field profile \#3. 


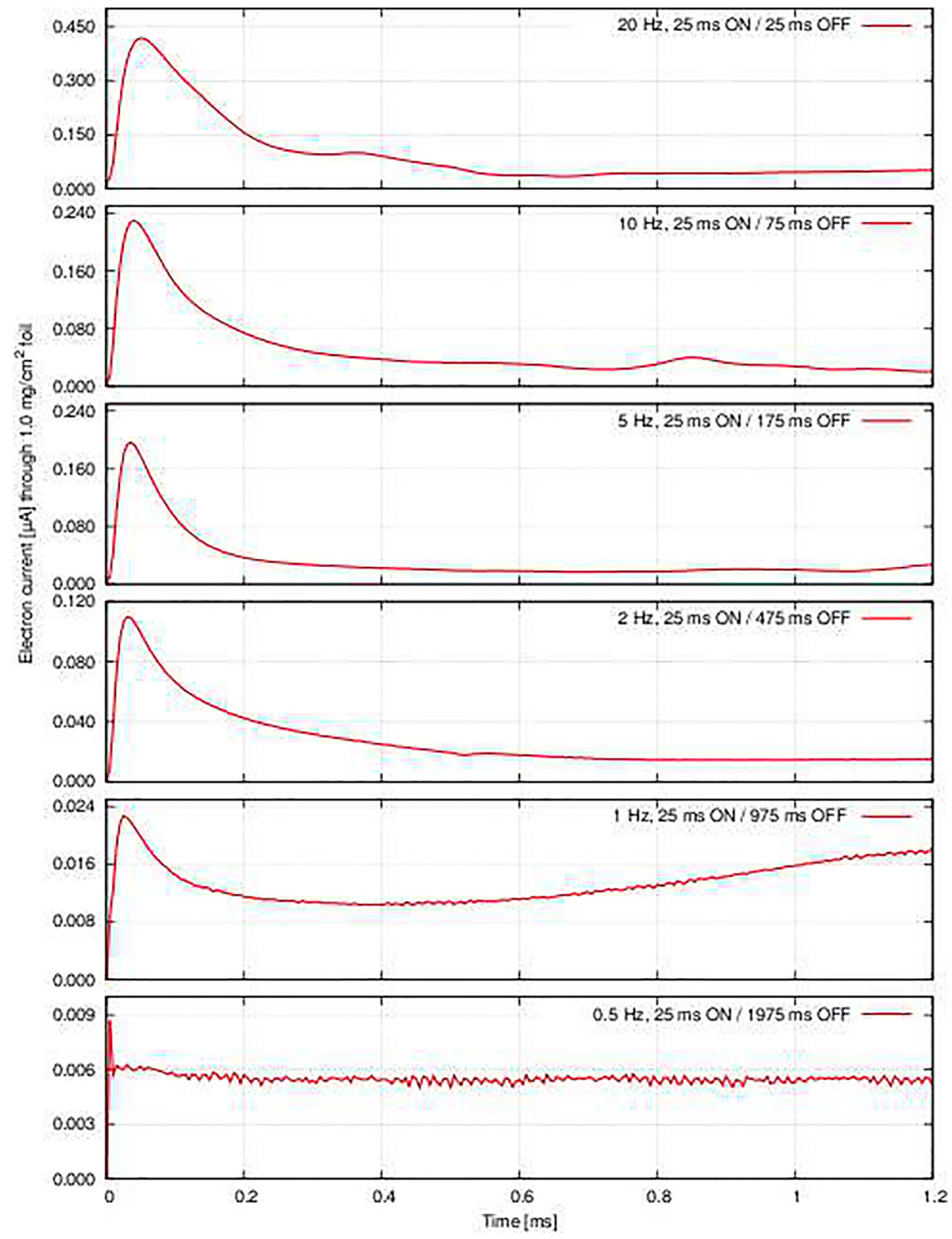

FIG. 7. Variation of the electron signal with a $25 \mathrm{~ms} / 700 \mathrm{~W}$ microwave pulse at different repetition rates at $\mathrm{B}$-field profile \#2. The measured results were obtained with a $1 \mathrm{mg} / \mathrm{cm}^{2}$ foil $(\mathrm{E}>20 \mathrm{keV})$.

seed electrons) or overlaps with the effects caused by the ionization cascade (high seed electron density).

The effect of the pulse repetition rate on electron signals in the beginning of the microwave pulse is presented in Fig. 7 showing an example measured with a $1 \mathrm{mg} / \mathrm{cm}^{2}$ foil (20 keV threshold) at $0.5-20 \mathrm{~Hz} / 25 \mathrm{~ms}$ pulses (note different vertical scales). The data were obtained with $700 \mathrm{~W}$ incident power and a solenoid field profile of $\mathrm{B}_{\mathrm{inj}}=2.01 \mathrm{~T} / \mathrm{B}_{\min }$ $=0.35 \mathrm{~T} / \mathrm{B}_{\text {ext }}=0.95 \mathrm{~T}$ (B-field profile \#2). The fact that the magnitude of the transient peak depends on the repetition rate suggests that the initial burst of electrons at the very beginning of the microwave pulse is indeed caused by the seed electrons that are expelled from the trap. A similar behavior depending on the pulse repetition rate was observed also with other aluminum foils.

In order to highlight the fact that the discussed burst of hot electrons is a transient, independent of the plasma breakdown, stepwise ionization, and gradual saturation of plasma density and temperature, Fig. 8 shows the electron signal measured with various foils, visible light signal recorded with the photodiode, and the total bremsstrahlung count rate for the full length $(25 \mathrm{~ms})$ of the microwave pulse. The presented data were measured with $200 \mathrm{~W}$ of incident power at $20 \mathrm{~Hz}$ repetition rate and a magnetic field configuration of $B_{i n j}$ $=1.95 \mathrm{~T} / \mathrm{B}_{\min }=0.32 \mathrm{~T} / \mathrm{B}_{\text {ext }}=0.90 \mathrm{~T}$ (B-field profile \#1).

The data show that with the given ion source settings, the initial transient peak is more pronounced with increasing electron energy, thus strengthening the claim that the rfinduced pitch angle scattering plays a significant role in determining the electron losses (as opposed to collisional scattering) of high energy electrons especially. Also, the bremsstrahlung count rate at energies $>35 \mathrm{keV}$ peaks immediately after microwave turn-on meaning that the burst of electrons is observed in both axial (electron detector) and radial (germanium detector) directions. The signals related to the existence of low energy electrons and their losses, i.e., electron flux measured without the aluminum foil and integrated light signal from the plasma, suggest that the EEDF in 


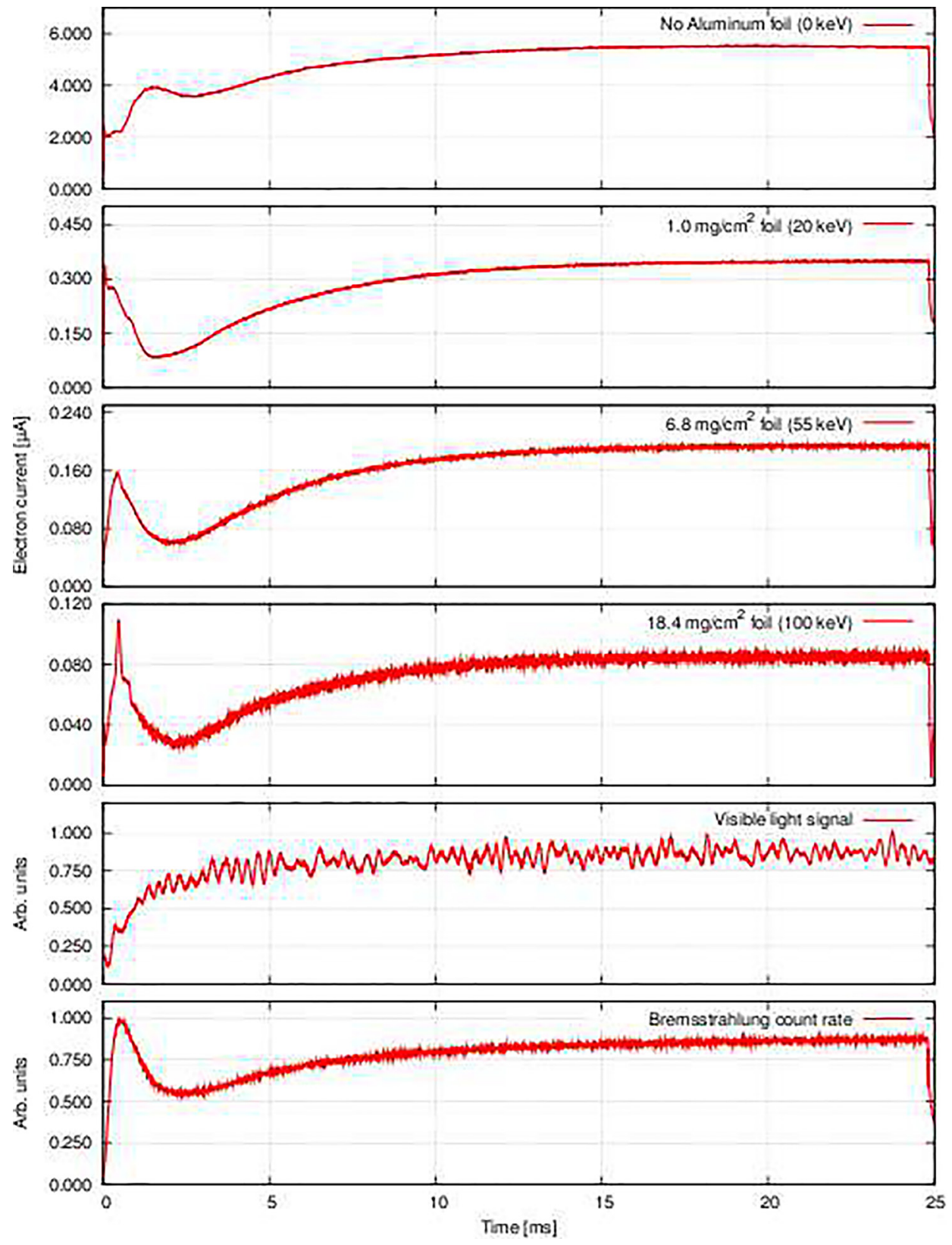

FIG. 8. Variation of the electron signal $(\mu \mathrm{A})$, visible light signal, and the total bremsstrahlung count rate with $25 \mathrm{~ms}$ $200 \mathrm{~W}$ microwave pulse, at $20 \mathrm{~Hz}$ repetition rate, and $\mathrm{B}$-field profile \#1, with the various foils. steady-state plasma differs greatly from the one observed during the burst. All these observations support the conclusion that at low plasma density in the beginning of the microwave pulse, the electric field and average electron energy are high, which enhances the microwave induced pitch angle scattering rate. As the ionization cascade proceeds, the electric field strength and average electron energy drop, ${ }^{20,26}$ which finally leads to saturation of e.g., plasma density and loss rates of electrons.

Table II summarizes the effects of magnetic field configuration and microwave power on the detected electron flux and bremsstrahlung count rate during the initial transient. The presented values are the ratios of the transient to the value of the signal at the end of the pulse with various repetition rates. The length of the microwave pulse was set to $25 \mathrm{~ms}$. With certain ion source settings, the signal to noise ratio was too low for the thickest two foils and, therefore, the corresponding data are omitted from the table.
The following is a summary of the qualitative tendencies observed with different ion source settings: (i) the electron flux during the transient burst increases with microwave power. This is probably due to increased plasma density during the microwave pulse, which in turn enhances the seed electron density in the beginning of the following pulse. Also, the transient could increase with power due to the fact that the RF-field strength scales with the power $(E \propto \sqrt{P})$. (ii) The electron flux during the transient burst increases with the pulse repetition rate, which can be explained by the higher seed electron density as well. (iii) Increasing the solenoid magnetic field strength results in s stronger burst of electrons (flux in axial direction) in the beginning of the microwave pulse. From the earlier discussion, it is known that the axial mirror ratio (best defined by $B_{\max } / B_{E C R}$ ) affects the hot electron confinement. During the microwave-off period, the electron loss rate depends on the strength of the mirror field and the rate of collisional scattering. The fact 
TABLE II. Variation of the ratio of electron signal $(\mu \mathrm{A})$ and total bremsstrahlung count rate at the initial transient to the value of the signal at the end of the microwave pulse, as a function of B-field configuration and microwave power, at different repetition rates.

\begin{tabular}{|c|c|c|c|c|c|}
\hline B-field profile & 1 & 2 & 3 & 2 & 2 \\
\hline$\mu$-wave power & $200 \mathrm{~W}$ & $200 \mathrm{~W}$ & $200 \mathrm{~W}$ & $500 \mathrm{~W}$ & $700 \mathrm{~W}$ \\
\hline Rep. rate $(\mathrm{Hz})$ & $2 / 5 / 10$ & $2 / 5 / 10$ & $2 / 5 / 10$ & $2 / 5 / 10$ & $2 / 5 / 10$ \\
\hline $\mathrm{e}^{-}$, all $\mathrm{E}$ & $0.12 / 0.20 / 0.26$ & $0.12 / 0.28 / 0.53$ & $3.16 / 2.28 / 1.87$ & $0.07 / 0.17 / 0.36$ & $0.07 / 0.12 / 0.22$ \\
\hline $\mathrm{e}^{-}, \mathrm{E}>20 \mathrm{keV}$ & $0.16 / 0.25 / 0.45$ & $1.04 / 1.79 / 7.24$ & $41.2 / 51.4 / 80.4$ & $0.76 / 2.08 / 3.98$ & $0.64 / 1.32 / 1.81$ \\
\hline $\mathrm{e}^{-}, \mathrm{E}>55 \mathrm{keV}$ & $0.07 / 0.20 / 0.37$ & $1.00 / 1.09 / 3.81$ & $40.1 / 45.4 / 42.9$ & $0.53 / 0.96 / 1.14$ & $0.51 / 0.57 / 0.78$ \\
\hline $\mathrm{e}^{-}, \mathrm{E}>100 \mathrm{keV}$ & $0.08 / 0.26 / 0.53$ & $1.28 / 1.42 / 2.11$ & $4.71 / 4.49 / 3.40$ & $0.55 / 1.01 / 1.67$ & $0.40 / 0.63 / 0.97$ \\
\hline $\mathrm{X}$-ray rate (ratio/norm.) $)^{34}$ & $1.14 / 0.41$ & $1.09 / 0.46$ & $1.33 / 1$ & $-/-$ & $-1-$ \\
\hline
\end{tabular}

that a stronger burst of electrons is observed with increasing B-field during the microwave-on period is probably due to a combination of two effects. First, the electron confinement is enhanced as a result of the higher axial mirror ratio during the microwave-off period which leads to a higher seed electron density in the beginning of the subsequent microwave pulse. Second, the lower average magnetic field gradient over the resonance zone leads to higher average electron energies and, thus lower collision frequency, as shown from bremsstrahlung measurements ${ }^{19}$ and direct measurement of the energy distribution of the escaping electrons. ${ }^{27}$ The lower gradient also enhances the energy gain of the electrons as shown by Eqs. (5) and (6) and presumably increases the pitch angle scattering rate in the beginning of the microwave pulse. The result suggests that the process responsible for the initial burst expels electrons to all directions, which is indicative for microwave induced pitch angle scattering. ${ }^{17}$ It is important to note that with this comparison, the initial electron burst was compared to the saturation electron flux at the same solenoid field setting and the behavior at different solenoid field settings was not compared. Furthermore, the observation of the transient peak in the beginning of the pulse, i.e., at low plasma density, across the given range of electron energies and ion source settings implies that the phase velocity of the microwave is significantly reduced, which highlights the importance of the resonant interaction inducing electron losses.

In addition to the initial electron burst, microwave induced electron losses presumably affect the decay of the electron flux following the trailing edge of the microwave pulse. Deviations from exponential afterglow decay (Coulomb collisions driving diffusion) could be interpreted as a signature of microwave induced pitch angle scattering actively increasing the electron loss rate during the microwave pulse. Figure 9 shows the electron signal measured with selected foils, visible light signal, total bremsstrahlung count rate, and microwave power signal associated with the trailing edge of the microwave pulse at $24.85 \mathrm{~ms}$. The presented example was measured with $200 \mathrm{~W}$ of incident power at $20 \mathrm{~Hz}$ repetition rate and a magnetic field configuration of $\mathrm{B}_{\text {inj }}=1.95 \mathrm{~T} / \mathrm{B}_{\min }=0.32 \mathrm{~T} / \mathrm{B}_{\text {ext }}=0.90 \mathrm{~T}$ (B-field profile \#1). The data were chosen for display because the signal levels are adequate and no instabilities affecting the electron losses are observed during the plasma decay, which is often the case. ${ }^{33}$

Independent of the foil thickness, the electron flux drops to less than $50 \%$ of its saturation value in $<20 \mu$ s after the microwave power is switched off, followed by slower decay lasting tens of milliseconds. In the case of the two thickest foils (not shown here), the signal practically vanishes immediately after the microwave is switch off. Also the bremsstrahlung count rate initially decays very fast and then follows a smoother, exponential behavior. On the contrary, the decay of the visible light signal, representing the excitation and recombination rate of low charge state ions, exhibits much slower decay. The microwave power signal decays from its maximum value to zero in less than $20 \mu \mathrm{s}$. The measured decay of the signal shown in Fig. 9 is limited by the RC-constant of the klystron output channel-the switch-off time of the RF-switch being $40 \mathrm{~ns}$ (from $100 \%$ to $10 \%$ ). Altogether, it is concluded that the escaping electron and bremsstrahlung signals decay faster than can be explained by Coulomb collisions alone. Thus, the data suggest that microwave induced pitch angle scattering contributes significantly (more than 50\%) to the electron losses across the whole energy range during the microwave pulse. The fractional contribution to electron losses due to the three loss mechanisms discussed in Sec. II has been numerically investigated by Cluggish et al. ${ }^{10}$ From the results of this simulation, it is concluded that Coulomb collision losses account for between $20 \%$ and $35 \%$ of the total electron losses under stable plasma conditions. The drop of the electron signal at the end of the microwave pulse to less than $50 \%$ of the rf ON signal suggests that the Coulomb collisions account for this fraction of the total losses, which is in good agreement with the numerical prediction.

The effect of ion source settings, i.e., microwave power and magnetic field configuration, on the decay characteristics of electron flux, bremsstrahlung count rate, and visible light signal is summarized in Table III, which shows each signal measured at $200 \mu \mathrm{s}$ and at $2 \mathrm{~ms}$ after the microwave switch off and normalized with respect to the steady-state value. It was observed that the repetition rate and duty factor did not affect the decay characteristics and, therefore, only data recorded with $20 \mathrm{~Hz} / 50 \%$ duty factor are displayed.

With all source settings, the qualitative decay characteristics of the electron, bremsstrahlung and visible light signals are similar to those illustrated in Fig. 9 i.e., the electron flux escaping the trap drops much faster than could be expected from diffusive decay. This corroborates the conclusion that during the microwave pulse, there is an active loss mechanism scattering electrons into the loss cone and contributing more than $50 \%$ to the total electron loss rate. 


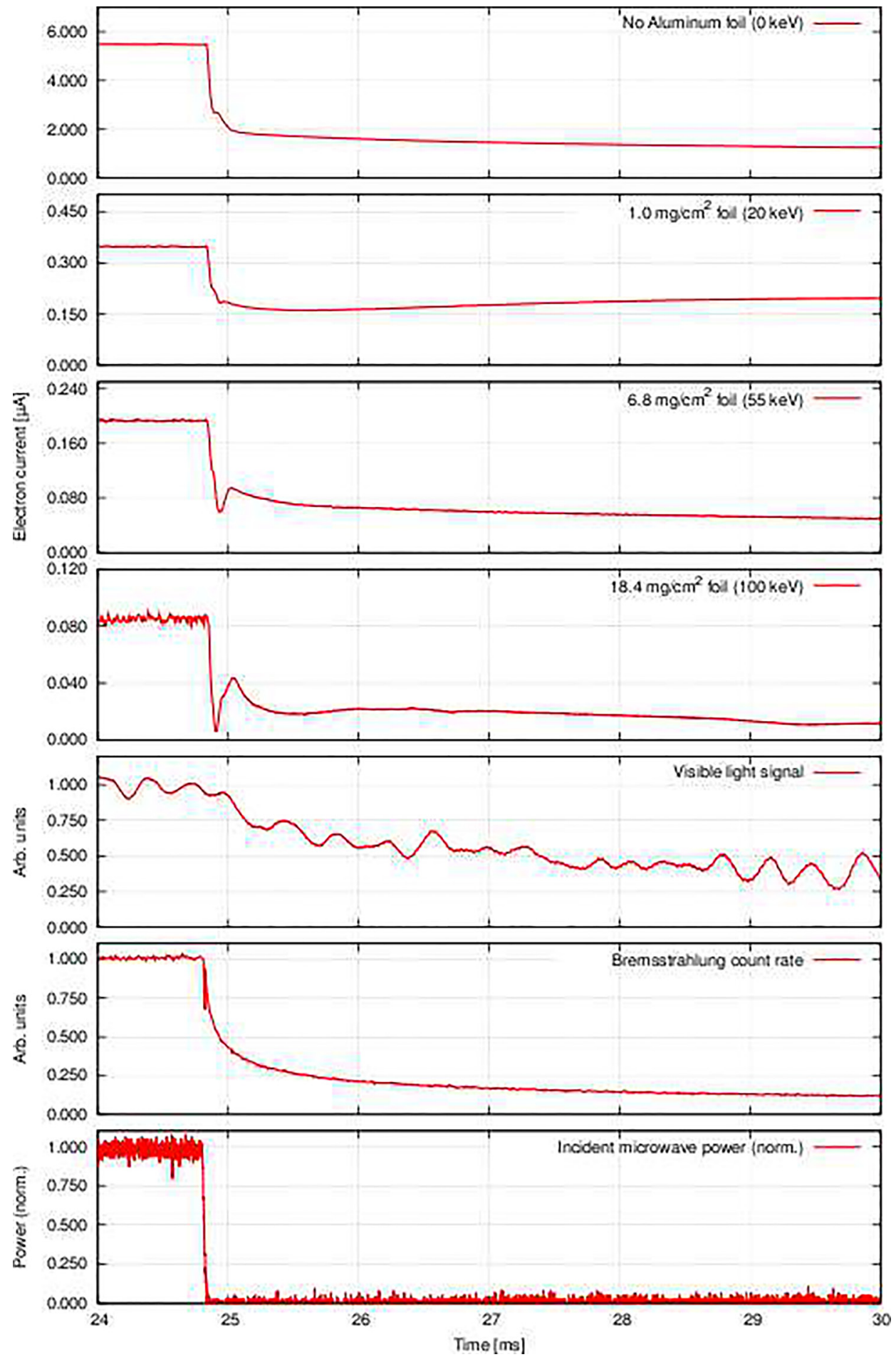

FIG. 9. Variation of the electron signal $(\mu \mathrm{A})$ (with selected foils), visible light signal, the total bremsstrahlung count rate, and the microwave power signal associated with the trailing edge of the microwave pulse at $24.85 \mathrm{~ms}$. The results displayed were taken with $200 \mathrm{~W}$ of incident power, at $20 \mathrm{~Hz}$ repetition rate, and B-field profile \#1.
TABLE III. Variation of the electron signal $(\mu \mathrm{A})$, total bremsstrahlung count rate, and the visible light signal measured at $200 \mu$ s and at $2 \mathrm{~ms}$ (after microwave switch off) and normalized with respect to the steady-state value, as a function of B-field configuration and microwave power.

\begin{tabular}{lccccc}
\hline \hline $\begin{array}{l}\text { B-field profile } \\
\mu \text {-wave power }\end{array}$ & $\begin{array}{c}\# 1 \\
200 \mathrm{~W}\end{array}$ & $\begin{array}{c}\# 2 \\
200 \mathrm{~W}\end{array}$ & $\begin{array}{c}\# 3 \\
200 \mathrm{~W}\end{array}$ & $\begin{array}{c}\# 2 \\
500 \mathrm{~W}\end{array}$ & $\begin{array}{c}\# 2 \\
700 \mathrm{~W}\end{array}$ \\
\hline $\mathrm{e}^{-}$, all E & $0.35 / 0.26$ & $0.31 / 0.24$ & $0.18 / 0.11$ & $0.23 / 0.21$ & $0.25 / 0.22$ \\
$\mathrm{e}^{-}, \mathrm{E}>20 \mathrm{keV}$ & $0.49 / 0.50$ & $0.36 / 0.71$ & $0.59 / 0.84$ & $0.50 / 0.92$ & $0.26 / 0.61$ \\
$\mathrm{e}^{-}, \mathrm{E}>55 \mathrm{keV}$ & $0.47 / 0.32$ & $0.24 / 0.64$ & $0.38 / 0.63$ & $0.61 / 0.60$ & $0.28 / 0.34$ \\
$\mathrm{e}^{-}, \mathrm{E}>100 \mathrm{keV}$ & $0.41 / 0.24$ & $-/-$ & $-/-$ & $0.47 / 0.41$ & $0.39 / 0.32$ \\
$\mathrm{X}-$ ray rate & $0.41 / 0.18$ & $0.37 / 0.21$ & $0.55 / 0.33$ & $-/-$ & $-/-$ \\
Visible light & & & & & \\
\hline \hline
\end{tabular}

\section{CONCLUSION}

The relative electron losses in $\mathrm{CW}$ operation and the temporal behavior of the electron flux escaping through the extraction mirror of an ECRIS in pulsed operation have been shown to exhibit features that can be associated with microwave induced pitch angle scattering. In the former case, it was observed that $r$ (defined above) increases with microwave power. An even more pronounced increase in $r$ at low plasma density with increasing microwave power was also observed. Both these behaviors can be attributed to microwave induced pitch angle scattering in action. With the latter case, the initial burst of electrons in the very beginning of 
the microwave pulse and the abrupt drop of electron flux at the trailing edge of the pulse is also suggested to be associated with microwave induced pitch angle scattering. Finally, it should be mentioned that the agreement between the experiment and simulations ${ }^{10}$ is good.

\section{ACKNOWLEDGMENTS}

V. Toivanen and J. Komppula are acknowledged for the technical assistance and analysis of the bremsstrahlung data acquired in the pulsed mode. M.S. wishes to thank Professor Paul Papka and the Department of Physics at the University of Stellenbosch for the use of their Silicon Drift Detector (X123-SDD) during the $\mathrm{CW}$ measurements described in this manuscript. The work of OT has been supported by the Väisälä Foundation of the Finnish Academy of Science and Letters. This research was conducted at JYFL and M.S. wishes to thank all those involved for their support. This work has been supported by the Academy of Finland under the Finnish Center of Excellence Program (Contract No. 213503) and mobility grant (No. 290390). The work is also based on the research supported in part by iThemba LABS and the National Research Foundation of South Africa (Grant Nos. 90741 and 109887). The work of V. Skalyga and I. Izotov was carried out with the support of the Federal Agency for Scientific Organizations in the frame of state order No. 0035-2014-0026.

${ }^{1}$ R. Geller, Electron Cyclotron Resonance Ion Sources and ECR Plasmas (Institute of Physics Publishing, Bristol, 1996).

${ }^{2}$ C. Barué, M. Lamoureux, P. Briand, A. Girard, and G. Melin, "Investigation of hot electrons in electron-cyclotron resonance ion sources," J. Appl. Phys. 76, 2662 (1994)

${ }^{3} \mathrm{G}$. Shirkov, "Multicomponent consideration of electron fraction of electron-cyclotron resonance source plasma," Rev. Sci. Instrum. 71, 850 (2000).

${ }^{4}$ C. Perret, A. Girard, H. Khodja, and G. Melin, "Limitations to the plasma energy and density in electron cyclotron resonance ion sources," Phys. Plasmas 6, 3408 (1999).

${ }^{5}$ C. C. Petty, D. L. Goodman, D. L. Smatlak, and D. K. Smith, "Confinement of multiply charged ions in an electron cyclotron resonance heated mirror plasma," Phys. Fluids B 3, 705 (1991).

${ }^{6}$ F. Jaeger, A. J. Lichtenberg, and M. A. Lieberman, "Theory of electron cyclotron resonance heating - I. Short time and adiabatic effects," Phys. Plasmas 14, 1073 (1972).

${ }^{7}$ I. B. Bernstein and D. C. Baxter, "Relativistic theory of electron cyclotron resonance heating," Phys. Fluids 24, 108 (1981).

${ }^{8}$ M. E. Mauel, "Electron-cyclotron heating in a pulsed mirror experiment," Phys. Fluids 27, 2899 (1984).

${ }^{9}$ J. Noland, O. Tarvainen, J. Benitez, D. Leitner, C. Lyneis, and J. Verboncoeur, "Studies of electron heating on a $6.4 \mathrm{GHz}$ ECR ion source through measurement of diamagnetic current and plasma bremsstrahlung," Plasma Sources Sci. Technol. 20, 035022 (2011).

${ }^{10} \mathrm{~B}$. Cluggish, L. Zhao, and J. S. Kim, "Modeling of the stability of electron cyclotron resonance ion source plasmas," Nucl. Instrum. Methods Phys. Res., Sect. A 631, 111 (2011).

${ }^{11}$ T. Thuillier, J. Angot, J. Y. Benitez, A. Hodgkinson, C. M. Lyneis, D. S. Todd, and D. Z. Xie, "Investigation on the electron flux to the wall in the VENUS ion source," Rev. Sci. Instrum. 87, 02A736 (2016).

${ }^{12}$ G. D. Shirkov, "A classical model of ion confinement and losses in ECR ion sources," Plasma Sources Sci. Technol. 4, 250 (1993).
${ }^{13}$ A. Girard, D. Hitz, G. Melin, and K. Serebrennikov, "Electron cyclotron resonance plasmas and electron cyclotron resonance ion sources: Physics and technology (invited)," Rev. Sci. Instrum. 75, 1381 (2004).

${ }^{14}$ A. Girard, C. Pernot, G. Melin, and C. Lécot, "Modeling of electron-cyclotron-resonance-heated plasmas," Phys. Rev. E 62, 1182 (2000).

${ }^{15}$ O. Tarvainen, J. Laulainen, J. Komppula, R. Kronholm, T. Kalvas, H. Koivisto, I. Izotov, D. Mansfeld, and V. Skalyga, "Limitations of electron cyclotron resonance ion source performances set by kinetic plasma instabilities," Rev. Sci. Instrum. 86, 023301 (2015).

${ }^{16}$ T. H. Stix, Waves in Plasmas (Springer-Verlag, New York, 1992).

${ }^{17}$ C. F. Kennel and F. Engelmann, "Velocity space diffusion from weak plasma turbulence in a magnetic field," Phys. Fluids 9, 2377 (1966).

${ }^{18}$ A. Girard, C. Perret, G. Melin, and C. Lécot, "Modeling of electron-cyclotronresonance ion source and scaling laws," Rev. Sci. Instrum. 69, 1100 (1998).

${ }^{19}$ T. Ropponen, P. Jones, T. Kalvas, H. Koivisto, P. Peura, O. Tarvainen, and P. Suominen, "Time evolution of endpoint energy of bremsstrahlung spectra and ion production from an electron cyclotron resonance ion source," in Proceeding of the 18th International Workshop on ECR Ion Sources TUCO-D01 (2008).

${ }^{20}$ T. Ropponen, O. Tarvainen, P. Suominen, T. K. Koponen, T. Kalvas, and H. Koivisto, "Hybrid simulation of electron cyclotron resonance heating," Nucl. Instrum. Methods Phys. Res., Sect. A 587, 115 (2008).

${ }^{21}$ M. C. Williamson, A. J. Lichtenberg, and M. A. Lieberman, "Self consistent electron cyclotron resonance absorption in a plasma with varying parameters," J. Appl. Phys. 72, 3924 (1992).

${ }^{22}$ H. Koivisto, P. Heikkinen, V. Hänninen, A. Lassila, H. Leinonen, V. Nieminen, J. Pakarinen, K. Ranttila, J. Ärje, and E. Liukkonen, "The first results with the new JYFL 14 GHz ECR ion source," Nucl. Instrum. Methods Phys. Res., Sect. A 174, 379 (2001).

${ }^{23}$ M. Sakildien, R. Kronholm, O. Tarvainen, T. Kalvas, P. Jones, R. Thomae, and H. Koivisto, "Inner shell ionization of argon in ECRIS plasma," Nucl. Instrum. Methods Phys. Res., Sect. A (published online).

${ }^{24}$ I. Izotov, D. Mansfeld, V. Skalyga, V. Zorin, T. Grahn, T. Kalvas, H. Koivisto, J. Komppula, P. Peura, O. Tarvainen, and V. Toivanen, "Plasma instability in the afterglow of electron cyclotron resonance discharge sustained in a mirror trap," Phys. Plasmas 19, 122501 (2012).

${ }^{25} \mathrm{See}$ http://www.nist.gov/pml/data/star/index.cfm for more information regarding the calculation of the range for electrons in aluminium.

${ }^{26}$ V. Toivanen, O. Tarvainen, C. Lyneis, J. Kauppinen, J. Komppula, and H. Koivisto, "ECRIS plasma chamber studies using a network analyzer as a loaded cavity probe,” Rev. Sci. Instrum. 83, 02 A306 (2012).

${ }^{27}$ I. Izotov, O. Tarvainen, V. Skalyga, D. Mansfeld, T. Kalvas, H. Koivisto, and R. Kronnholm, "Measurement of the energy distribution of electrons escaping minimum-B ECR plasmas," Phys. Plasmas 27(2), 025012 (2018).

${ }^{28}$ S. Gammino, D. Mascali, L. Celona, F. Maimone, and G. Ciavola, "Considerations on the role of the magnetic field gradient in ECR ion sources and build-up of hot electron component," Plasma Sources Sci. Technol. 18, 045016 (2009).

${ }^{29}$ H. Koivisto, "The effect of microwave frequency and grad B on the energy of electrons in an electron cyclotron resonance ion source," Rev. Sci. Instrum. 70, 2979 (1999).

${ }^{30} \mathrm{~W}$. Lotz, "Electron-impact ionization cross-section and ionization rate coefficients for atoms and ions from scandium to zinc,” Z. Phys. 220, 466 (1969).

${ }^{31}$ O. Tarvainen, P. Suominen, and H. Koivisto, "A new plasma potential measurement instrument for plasma ion sources," Rev. Sci. Instrum. $\mathbf{7 5}$, 3138 (2004).

${ }^{32}$ O. Tarvainen, T. Ropponen, T. Thuillier, J. Noland, V. Toivanen, T. Kalvas, and H. Koivisto, "The role of seed electrons on the plasma breakdown and preglow of electron cyclotron resonance ion source," Rev. Sci. Instrum. 81, 02A303 (2010).

${ }^{33}$ D. Mansfeld, I. Izotov, V. Skalyga, O. Tarvainen, T. Kalvas, H. Koivisto, J. Komppula, R. Kronholm, and J. Laulainen, "Dynamic regimes of cyclotron instability in the afterglow mode of minimum-B electron cyclotron resonance ion source plasma," Plasma Phys. Controlled Fusion 58, 045019 (2016).

${ }^{34}$ Measured at $20 \mathrm{~Hz}$ repetition rate. Ratio = count rate during the initial transient/count rate at $25 \mathrm{~ms}$. Norm. $=$ count rate normalized to maximum at $25 \mathrm{~ms}$ with different B-field configurations.

${ }^{35}$ Due to some ripple, the visible light signals have (estimated) error bars of $\pm 0.05$ 